\title{
MODELO COMPUTACIONAL PARA REVISÃO SISTEMÁTICA BASEADO NA TEORIA DE REDE COMPLEXA
}

Jótelly Barros Oliveira ${ }^{1}$; Renata Souza Freitas Dantas Barreto 2; Edvaldo Melquiades Ribeiro do Bonfim Júnior 3; Tereza Kelly Gomes Carneiro 4; Hernane Borges de Barros Pereira 5; Ingrid Winkler 6; Roberto Luiz Souza Monteiro ${ }^{7}$

1 Centro Universitário SENAI CIMATEC, Salvador, BA, jotelly@gmail.com;

${ }^{2}$ Centro Universitário SENAI CIMATEC, Salvador, BA

${ }^{3}$ Centro Universitário SENAI CIMATEC, Salvador, BA

${ }^{4}$ IFBA - Instituto Federal de Educação, Ciência e Tecnologia da Bahia, Salvador, BA

${ }^{5}$ Centro Universitário SENAI CIMATEC, Salvador, BA

${ }^{6}$ Centro Universitário SENAI CIMATEC, Salvador, BA

${ }^{7}$ Centro Universitário SENAI CIMATEC, Salvador, BA

Resumo: Para se realizar uma pesquisa científica é necessário a priori uma revisão sistemática (RSL) do tema escolhido para legitimar os estudos futuros. O processo de uma RSL demanda tempo e exige minuciosidade. O objetivo deste trabalho é apresentar um modelo computacional (MC) para revisão sistemática baseado na teoria de redes complexas, especificamente, rede de citação sobre odometria visual. O nosso modelo computacional é baseado (1) nos índices de redes, (2) aplicação de um algoritmo para automatizar o processo de conversão do banco de dados, (3) visualização e análise dos dados. Analisamos por meio do modelo computacional 2462 periódicos e verificamos quais os artigos que estão em voga ou no mínimo são mais citados na área de estudo escolhida, neste caso, odometria visual [1].

Palavras-Chave: redes complexas; rede de citação; revisão sistemática; modelo computacional; odometria visual.

\section{COMPUTER MODEL FOR SYSTEMATIC REVIEW BASED ON COMPLEX NETWORK THEORY}

Abstract: To carry out a scientific research it is necessary a priori a systematic review (RSL) of the chosen theme to legitimize future studies. The process of an RSL takes time and requires thoroughness. The aim of this paper is to present a computational model (MC) for systematic review based on the theory of complex networks, specifically, citation network on visual odometry. Our computational model is based on (1) network indices, (2) application of an algorithm to automate the database conversion process, (3) data visualization and analysis. Through the 2462 periodical computational model, we analyzed which articles are in vogue or at least are most cited in the chosen study area, in this case, visual odometry [1].

Keywords: complex networks; citation network; systematic review; computational model; visual odometry.

\section{INTRODUÇÃO}


Quando optamos em realizar uma pesquisa científica surge a necessidade de uma revisão sistemática da literatura (RSL) do tema escolhido para legitimar os estudos futuros. O processo de uma RSL demanda tempo e exige minuciosidade. $O$ objetivo deste trabalho é apresentar um modelo computacional para revisão sistemática baseado na teoria de redes complexas, especificamente, rede de citação.

Escolhemos para a elaboração do banco de dados o tema odometria visual [1] pela relevância da temática aos interesses dos autores desta pesquisa. Em tempo, demonstraremos a eficiência do modelo computacional na obtenção e filtragem dos dados em questão, gerando as informações inerentes a rede de citação do tema proposto, este processo poderá ser utilizado para qualquer tema que tenha como objetivo o desenvolvimento de uma rede de citações baseadas nos assuntos escolhidos.

O nosso modelo computacional é baseado (1) nos índices de redes, (2) aplicação de um algoritmo para automatizar o processo de conversão do banco de dados, (3) visualização e análise dos dados. Foi possível averiguar por meio do modelo computacional quais são os periódicos que estão em voga ou no mínimo são mais citados na área de odometria visual. O Modelo se mostrou eficiente auxiliando a RSL.

Redes complexas referem-se a um sistema que apresenta vértices (ou nós) que são conectados por meio de arestas [2] normalmente representadas por grafos, esses sistemas possuem entidades ou vértices que compõem o conjunto denotado por $V(G)$ e as arestas são representadas por $E(G)$ [3] que matematicamente, os grafos são descritos da seguinte forma $G=(V ; E) V$ finito e não vazio e, $E$ a relações binárias sobre V.

A pesquisa sobre redes de citação advém de um estudo mais específico sobre a teoria de redes complexas. Uma das relevâncias que uma rede de citação possui é sua capacidade em apresentar especialmente como o conhecimento é compartilhado e difundido entre atores que efetivamente o usaram.

Neste contexto o objetivo deste trabalho é apresentar um modelo computacional para revisão sistemática baseado na teoria de redes complexas, utilizando de forma ilustrativa o tema odometria visual proposto para mostrar o potencial deste modelo.

O trabalho está organizado em cinco seções. Além desta Introdução, a seção 2 descreve a metodologia utilizada na investigação, a seção 3 aborda os resultados e discute-os, e seção 4 são apresentadas as conclusões e indicações de pesquisas futuras.

\section{METODOLOGIA}

Nesta seção, explanaremos as etapas que abrangem o método proposto para realizar a RSL e a rede de citação, amostragem e seus critérios, e os procedimentos para a construção das redes. 


\subsection{Modelo computacional baseado em redes de citação}

Para esta pesquisa utilizamos como base de dados o portal de periódicos IEEE Xplore Digital Library [5], utilizando como string de busca [((((("Abstract": "Visual odometry") OR "Abstract":"Egomotion") OR "Abstract":"Computer Vision") OR "Abstract":" Robot vision") OR "Abstract":"SLAM") AND "Abstract":"Underwater")], incluindo as palavras-chaves (Visual odometry, Egomotion, Computer Vision, Robot vision, SLAM e Underwater), obtendo-se assim 318 resultados.

Optamos em delimitar o tema com um intervalo de tempo entre 2013-2019, com o intuito de abranger resultados mais recentes, sendo assim, retornaram 172 artigos principais (AP) válidos para a elaboração do banco de dados. O processo metodológico abordado nesta pesquisa, possui cinco etapas e seis processos ilustradas na Figura 1.

Figura 1: Fluxograma da metodologia Modelo computacional para rede de citação.

Fonte: Autores (2019)

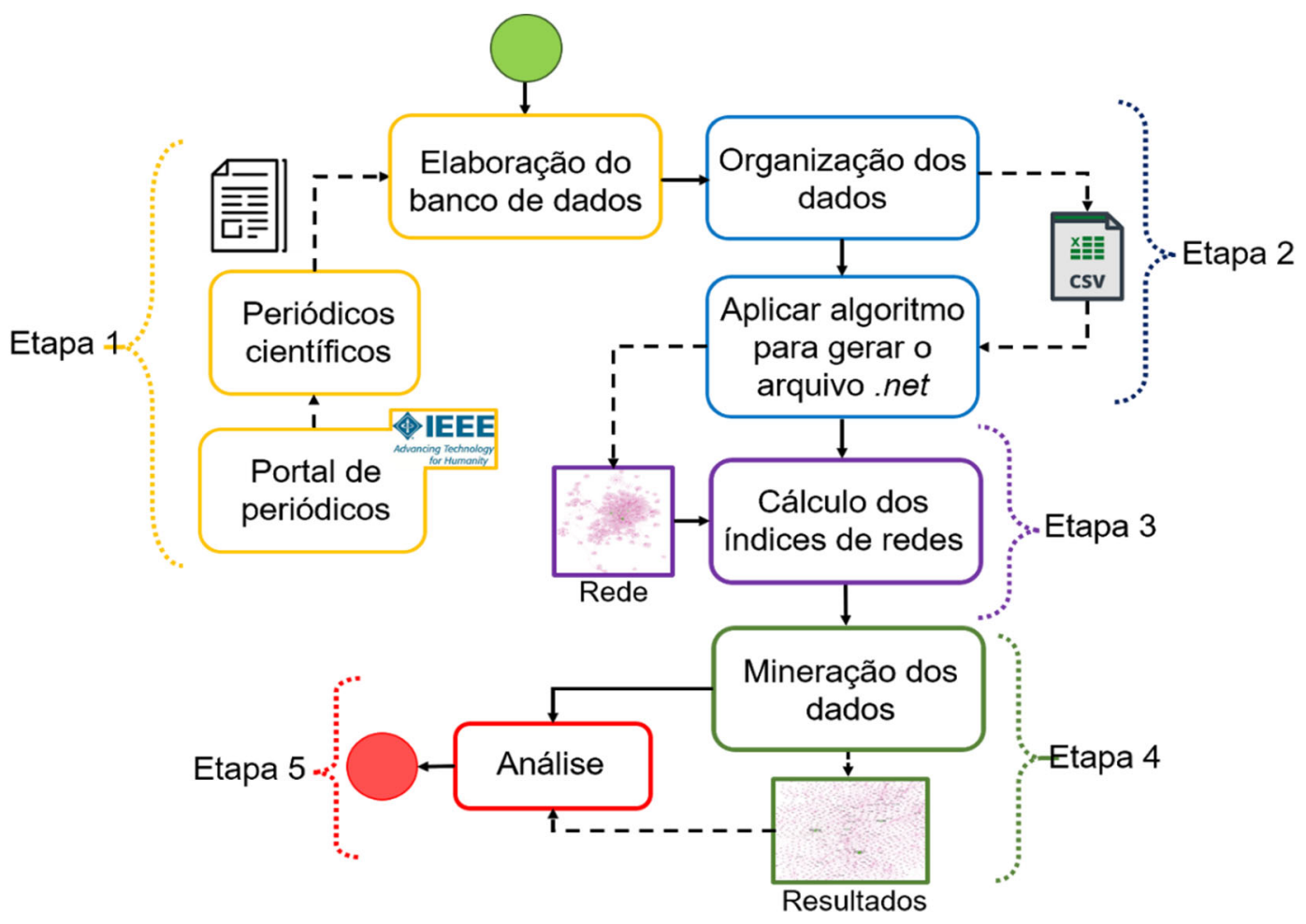

Na Etapa 1 (cor laranja da Figura 1) a metodologia do trabalho envolve o processo: (1) a elaboração do banco de dados com o tema escolhido para o estudo, incluindo a escolha dos portais de periódicos para a obtenção dos artigos (e.g IEEE Institute of Electrical and Electronic Engineers).

Na Etapa 2 (cor azul da Figura 1) após a escolha do portal (ou portais) de periódicos e coleta dos artigos, é necessário realizar o processo de organização dos dados na planilha contendo os seguintes campos: Código de artigos (código 
incremental P000 respectivo para cada um dos títulos), "ano", "título", "ISSN", "autor", "mês", "DOI", "palavras-chave" e "resumo".

Em seguida deve-se criar uma nova planilha com os dados referentes ao Artigo principal (AP) e Artigo Citado (AC) sendo eles: Id (código incremental 000), "Código_AP", "Titulo_AP", "Ano_AP", "Código_AC", "Titulo_AC" e "Ano_AC", classificado em ordem alfabética ('A' $<$ ' $Z$ ') o campo "Titulo de artigos". Com os dados organizados realizou-se o processo de construção da rede. Desenvolvemos um algoritmo, para fazer o tratamento final dos dados e gerar um arquivo de rede para ser visualizado em no software de visualização de redes, neste caso, o Gephi 0.92.

Na Etapa 3 (cor roxa da Figura 1), calculou-se as propriedades da rede, grau, modularidade, coeficiente de aglomeração. Para finalizar a Etapa 3 é importante usar algoritmos de distribuição espacial para melhor visualizar os vértices ou arestas desejadas.

A Etapa 4 (cor verde da Figura 1) representa o momento de mineração e refinamento dos dados para gerar a rede final que na próxima etapa será analisada.

Por último, a Etapa 5 (cor vermelha da Figura 1) consiste na verificação dos resultados e inferências do analista de forma mais conclusivas.

\section{RESULTADOS E DISCUSSÃO}

Nesta seção, explanaremos de maneira concisa uma possível análise que o modelo computacional baseado em redes de citação pode proporcionar.

\subsection{Análise para revisão sistemática utilizando MCRC}

Após a realização dos procedimentos citados na Seção 2, obtivemos uma rede com um total de 2462 (AP e AC) vértices e 3358 arestas, dentre os índices possíveis a serem calculados, optamos em analisar o grau de entrada, que nos diz sobre quantas vezes um artigo foi citado entre seus pares. Vejamos na Figura 2:

Figura 2: Rede total com 2462 vértices (artigos) e 3348 arestas (citações) sobre Odometria Visual

Fonte: Autores (2019) 


\section{CIRCULAR ECONOMY ECONOMIA CIRCULAR}

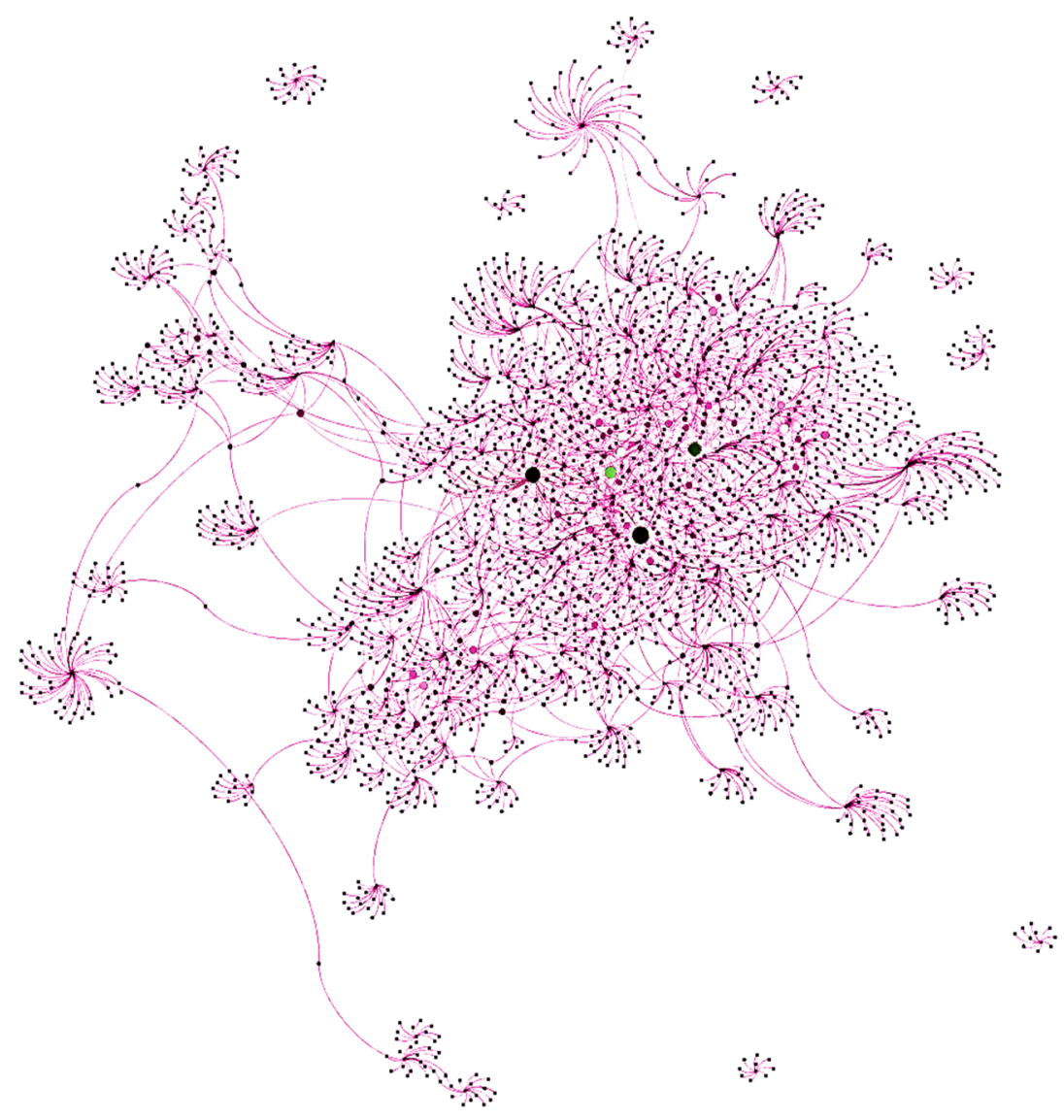

Os pontos mais escuros na rede referem-se aos periódicos mais citados, o artigo que possui o maior grau de entrada (23) é o "PC787" (Distinctive image features from scale-invariant keypoints), isto quer dizer que ele é citado por 23 artigos, o segundo artigo com grau de entrada (20) é o "PC1739" (Realtime visual slam for autonomous underwater hull inspection using visual saliency). Na Tabela 1 podemos verificar quais os 10 artigos mais citados entre os 2462 .

Tabela 1: Os 10 artigos com maior grau de entrada com seus códigos e títulos correspondentes.

Fonte: Autores (2019)

\begin{tabular}{c|c|c} 
Código & Título & $\begin{array}{c}\text { Grau de } \\
\text { Entrada }\end{array}$ \\
\hline PC787 & $\begin{array}{c}\text { Distinctive image features from } \\
\text { scale-invariant keypoints }\end{array}$ & 23 \\
\hline PC1739 & $\begin{array}{c}\text { Realtime visual slam for autonomous underwater hull } \\
\text { inspection using visual saliency }\end{array}$ & 20 \\
\hline PC1204 & Isam incremental smoothing and mapping & 17 \\
\hline
\end{tabular}




\begin{tabular}{c|c|c} 
PC276 & $\begin{array}{c}\text { Advanced perception navigation and planning for autonomous } \\
\text { in-water ship hull inspection }\end{array}$ & 15 \\
\hline PC2066 & Surf speeded up robust features & 13 \\
\hline PC435 & Auv navigation and localization a review & 12 \\
\hline PC1948 & Simultaneous localization and mapping part I & 12 \\
\hline PC1960 & Single image haze removal using dark channel prior & 12 \\
\hline PC1667 & Probabilistic robotics & 11 \\
\hline PC1137 & $\begin{array}{c}\text { Imaging sonar-aided navigation for autonomous underwater } \\
\text { harbor surveillance }\end{array}$ & 11
\end{tabular}

\section{CONCLUSÃO}

Este trabalho teve como objetivo apresentar um modelo computacional para revisão sistemática baseado na teoria de redes complexas, utilizando de forma ilustrativa o tema odometria visual proposto para mostrar o potencial deste modelo.

Diante do exposto ao longo deste trabalho, é possível concluirmos que o nosso modelo computacional mostrou-se eficiente no auxílio da elaboração de uma revisão sistemática, por demonstrar de forma assertiva quais trabalhos mais citados na janela de tempo pré-definida. É correto afirmar, que esta informação a respeito dos periódicos, serve como direcionamento para um início de uma pesquisa científica.

Como pesquisas futuras, sugerimos a elaboração de uma rede de coautoria e de títulos visando contribuir para o processo de mineração de dados, nas redes semânticas e para mapeamento e representação do conhecimento.

\section{Agradecimentos}

Os autores agradecem ao grupo de pesquisa em Realidade Aumentada e Realidade Virtual para Inovação na Indústria, Saúde e Educação (CNPq), grupo de Pesquisa Fuxicos e Boatos (CNPq), grupo de pesquisa TICASE (CNPq), a EMBRAPII pelo auxílio financeiro da bolsa de formação e desenvolvimento tecnológico concedida e o apoio financeiro da FAPESB por meio da bolsa de doutorado No BOL0239/2018.

\section{REFERÊNCIAS}

1SCARAMUZZA, Davide; FRAUNDORFER, Friedrich. Visual odometry [tutorial]. IEEE robotics \& automation magazine, v. 18, n. 4, p. 80-92, 2011.

3BARABASI, A. Network Science. United Kingdom: Cambridge, 2016. 
${ }^{4}$ GROSS, J. YELLEN, J. Graph theory and its applications. Boca Raton: CRC Press, 1999.

${ }^{5}$ IEEEXPLORE, IEEE Xplore Digital Library. Disponível em: <https://ieeexplore.ieee.org/Xplore/home.jsp>. Acesso em 16 de setembro de 2019. 